\title{
Adenoid cystic carcinoma of the Bartholin's gland: the University of Michigan experience
}

\author{
R. J. LELLE, K. P. DAVIS \& J. A. ROBERTS
}

Division of Gynecologic Oncology, Department of Obstetrics and Gynecology, The University of Michigan, Ann Arbor, Michigan, USA

\begin{abstract}
Lelle RJ, Davis KP, Roberts JA. Adenoid cystic carcinoma of the Bartholin's gland: the University of Michigan experience. Int J Gynecol Cancer 1994, 4, 145-149.

Adenoid cystic carcinoma of the Bartholin's gland has been encountered in 11 patients at the University of Michigan Medical Center since 1936. At the time of presentation the average age was 48.9 years, the lesion size was between 0.5 to $4 \mathrm{~cm}$. The presenting symptoms were pain and/or pruritis associated with a solitary mass. Early in this series, excisional biopsy was used to treat eight patients. The last three patients have been treated with a radical vulvectomy and unilateral or bilateral groin lymph node dissection. Local recurrence has occurred in five patients and distant recurrence in four patients. In spite of the high recurrence rate, 5and 10-year survival has been high with all seven evaluable patients surviving 5 and 10 years. However, adenoid cystic carcinoma of the vulva is associated with late recurrences and metastases: three patients were dead of disease at 12, 15 and 31 years after initial diagnosis.
\end{abstract}

KEYWORDS: adenoid cystic carcinoma, Bartholin's gland, vulvar malignancy.

Vulvar carcinoma is the fourth most common female genital tract malignancy. Among vulvar neoplasms, an estimated 5\% are of Bartholin's gland origin. Adenocarcinoma or squamous cell carcinoma account for 90\% of these Bartholin's gland neoplasms. The remaining types include adenoid cystic carcinomas, melanomas, sarcomas, and undifferentiated neoplasms. Adenoid cystic carcinoma histologically exhibits a classical cribriform arrangement of tubal and glandular elements. Perineural and lympathic invasion is characteristic of this tumor, and may explain its propensity for causing pain and recurrence.

Copeland et al. reported ${ }^{(1)}$ five patients with primary

Address for correspondence: James A. Roberts, MD, Director, Division of Gynecologic Oncology, Department of Obstetrics and Gynecology, University of Michigan, Ann Arbor, Michigan 481090718, USA. adenoid cystic carcinoma of the Bartholin's gland, and reviewed 32 additional cases from the literature. Overall, long-term survival was excellent. However, there was a propensity for frequent ipsilateral vulvar recurrence. This is in contrast to the more common extragenital adenoid cystic carcinoma which lends itself to adequate therapy and a long disease free interval.

This study was undertaken to evaluate our experience at the University of Michigan Medical Center with this rare genital malignancy.

\section{Patients and methods}

A review of the UMMC Tumor Registry was undertaken to identify all patients with a diagnosis of adenoid cystic carcinoma of the Bartholin's gland seen 
between 1936 and 1991 . The criteria for the diagnosis of primary Bartholin's gland carcinoma include: tumor involving the area of the Bartholin's gland, histologic area of transition from normal to neoplastic elements, and no other primary carcinoma present ${ }^{(2)}$.

Pathologic slides from patients were reviewed at the Gynecologic Tumor Conference to confirm the diagnosis of adenoid cystic carcinoma. Patient records were reviewed for clinical information, and follow-up was available through the Tumor Registry.

\section{Results}

Eleven patients with adenoid cystic carcinoma of the Bartholin's gland were identified (Table 1). Four of these patients were reported previously by $\mathrm{Abell}^{(3)}$. The most common presenting symptoms were pain and/or pruritis associated with the physical finding of solitary mass in the region of the Bartholin's gland. The median age at diagnosis was 48.9 years (range from 38 to 63 years). The size of the primary lesion ranged from 0.5 to $4 \mathrm{~cm}$. The initial therapeutic approach included excisional biopsy, i.e. resection of the visible lesion with minimal margins $(n=7)$, partial vulvectomy $(n=1)$, and radical vulvectomy with groin lymph node dissection $(n=3)$. Follow-up data were available for nine patients. The time of observation ranged from one to 31 years (median 12 years).

Five of the eleven patients developed local recurrence with a median progression-free interval of 8 years (range 2-13 years). Distant recurrence was diagnosed in four patients after 11, 15 and 16 years. The site of distant recurrence included lung, bone, liver, and lymph nodes. One patient (\#4) had isolated pulmonary metastases. Another patient, (\#7) showed lung and pubic bone involvement with positive pelvic lymph nodes, while local bone metastases (ilium and femoral head) were confirmed for a third patient (\#6). Skeletal metastases outside the pelvis were not identified in any of these ten patients. One patient (\#8) developed both lung and liver metastases. Six out of the eleven patients in this series have died. Three patients died from systemic spread of the vulvar malignancy. Two patients died of causes not related to the vulvar malignancy and in one patient the cause of death is unknown. All three patients who died from the disease had developed local recurrences with subsequent pulmonary metastases, in two patients in conjunction with spread to bone (\#7) and liver (\#8), respectively.

Five patients had disease to surgical margins, and one of these developed a local recurrence. Of the four patients who had negative margins, all four developed a recurrence.

\section{Discussion}

Five of the 11 patients presented in this report developed local recurrence. There was a long interval between the initial diagnosis of the disease and progression. The median time interval of eight years reported here agrees with that summarized in the literature ${ }^{(1)}$. There also appears to be a propensity for local recurrences prior to the development of distant metastases. Copeland et al..$^{(1)}$ and Bernstein et al. ${ }^{(4)}$ point out, that the most important aspect of treatment is to obtain clear surgical margins. However, in our review of the eleven patients treated at the University of Michigan Medical Center, the local recurrence rate did not appear to be influenced by the presence or absence of disease at the surgical margins. All four patients who had negative margins at the time of primary surgery developed a local vulvar recurrence. This could be due to early local dissemination of tumor cells beyond the margin of resection. In adenoid cystic carcinomas, the tumor grows along the nerve roots, and this might not be recognized histologically. In contrast, only one of the five patients with positive margins (\#7) developed a recurrence after a diseasefree interval of eight years. It can be speculated that the follow-up intervals for three of the remaining four patients, who had positive margins at the time of first treatment, were too short to let a local recurrence become evident: one of these patients (\#3) was from the earlier series and was lost to follow-up after 3 years, and the other two patients (\#10 and \#11) were diagnosed more recently (in 1985 and 1989).

The above observations seem to suggest that eventually all patients will develop a recurrence regardless of resection margin at the time of initial therapy. However, in some patients the disease-free interval will be longer than the natural life expectancy, so that the patient will die from causes other than the recurrent vulvar malignancy.

The number of patients in this report is small and statistically reliable data on the natural history of adenoid cystic carcinoma of the vulva are not available. Therefore, the necessity of a wide local resection to obtain free margins should not be dismissed. However, radical resections with considerable morbidity do not appear to be justified either. These should be reserved for the larger lesions or when disease is expected to extend into the ischiorectal fossa.

Fifteen patients reported in the literature have un- 
dergone groin node dissections and only two were found to have metastatic disease ${ }^{(1)}$. Both of these were located on the ipsilateral side of the vulvar neoplasm. One of the patients reported here (\#7) developed positive pelvic lymph nodes. However, these were not diagnosed until 11 years after the primary therapy at the time of a second recurrence. None of the three patients who underwent unilateral or bilateral groin lymph node dissection had positive nodes. The limited evidence that is available in the literature ${ }^{(1)}$ suggests that a groin lymph node dissection can be limited to an ipsilateral dissection as the disease has not yet been reported to involve the contralateral lymph nodes. With only $10 \%$ of lymph nodes positive, the dissection may be reserved for those patients felt to be at high risk for nodal involvement.

The metastatic spread of this neoplasm appears to occur by the hematogenous and geographic route. The hematogenous pathway appears a likely mechanism for those patients with lung and bony metastasis, whereas direct extension along the neural sheath may be responsible for frequent local recurrences. This spread pattern appears similar to that of primary adenoid cystic carcinoma from other anatomic sites, such as the glands of the neck ${ }^{(5)}$, upper respiratory tract $^{(6)}$, and breast ${ }^{(7)}$.

Chemotherapy has been utilized for metastatic adenoid cystic carcinoma of the salivary ${ }^{(5)}$ and parotid gland $^{(8)}$. There is little information available on its use in the female genital tract. One patient (\#8), received cyclophosphamide as an adjuvant therapy after wide excision of a recurrence in the labium. She died 15 years after initial diagnosis from wide-spread metastatic disease. Other reports of chemotherapeutic treatment of metastatic adenoid cystic carcinoma of the Bartholin's gland include a combination of methotrexate, Dactinomycin, and chlorambucil ${ }^{(9)}$ or doxorubicin therapy ${ }^{(10)}$. Further evaluation of chemotherapy regimens for treatment of recurrent diseases are needed to evaluate its efficacy for known metastasis.
However, due to the extended interval to recurrence it will be difficult to show any benefit for this adjuvant therapy.

Copeland et al..$^{(1)}$ reported a survival rate of 71 and $59 \%$ for 5 and 10 years, respectively. In the present series 5- and 10-year survival was $100 \%$, as the three patients who died of the disease lived for 12,15 and 31 years after the initial diagnosis. The disease-free interval for the patients in Copeland's series was $47 \%$ at 5 years and $38 \%$ at 10 years as compared with $83 \%$ (five out of six patients) and 33\% (two out of six patients) in the present study.

\section{References}

1 Copeland LJ, Sniege N, Gershenson DM, Saul PB, Stringer CA, Seski JC. Adenoid cystic carcinoma of the Bartholin gland. Obstet Gynecol 1986; 67: 115-20.

2 Chamlian DL, Taylor HB. Primary carcinoma of the Bartholin's gland: a report of 24 patients. Obstet Gynecol 1972; 39: 489-94.

3 Abell MR. Adenocystic (pseudoadenomatous) basal cell carcinoma of vestibular glands of vulva. Am J Obstet Gynecol 1963; 86: 470-82.

4 Bernstein SG, Voet RL, Lifshitz S, Buchsbaum HJ. Adenoid cystic carcinoma of Bartholin's gland. Am J Obstet Gynecol 1983; 147: 385-90.

5 Budd GT, Groppe CW. Adenoid cystic carcinoma of the salivary gland. Cancer 1983; 51: 589-90.

6 Marsh WL, Allen MS. Adenoid cystic carcinoma: biologic behavior in 38 patients. Cancer 1979; 43: 1463-73.

7 Peters GN, Wolff M. Adenoid cystic carcinoma of the breast: report of 11 new cases: review of the literature and discussion of biologic behavior. Cancer 1982; 52: 680-6.

8 Skibba JL, Hurley JD, Ravelo HV. Complete response of a metastatic adenoid cystic carcinoma of the paratoid gland to chemotherapy. Cancer 1981; 47: 2543-8.

9 Eichner E. Adenoid cystic carcinoma of the Bartholin gland. Obstet Gynecol 1963; 21: 608-13.

10 Wheelock JB, Goplerud DR, Dunn LJ, Oates JF. Primary carcinoma of the Bartholin gland: a report of ten cases. Obstet Gynecol 1984; 63: 820-4.

Accepted for publication 14 July 1993 
I48 R. J. Lelle et al.

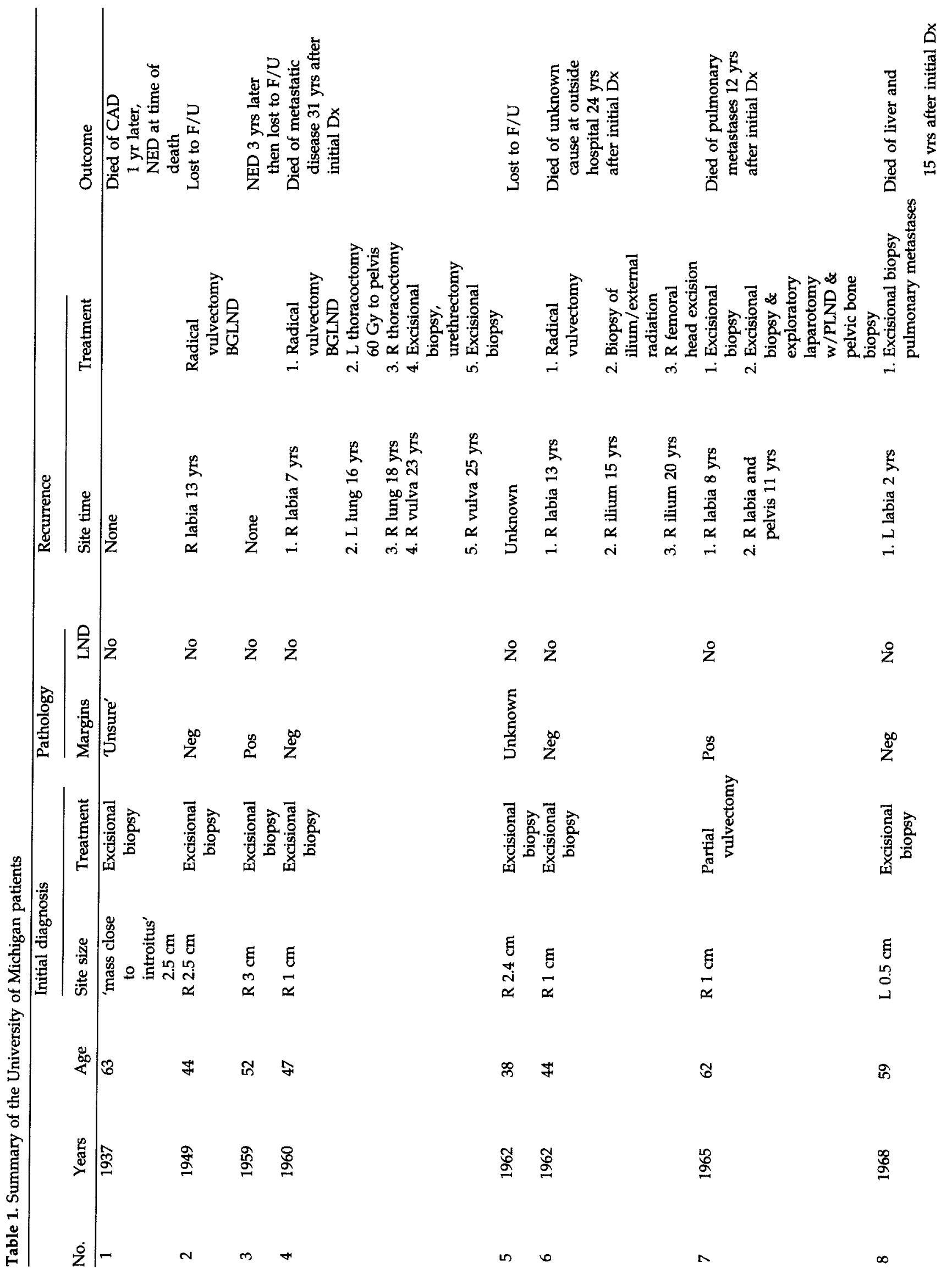



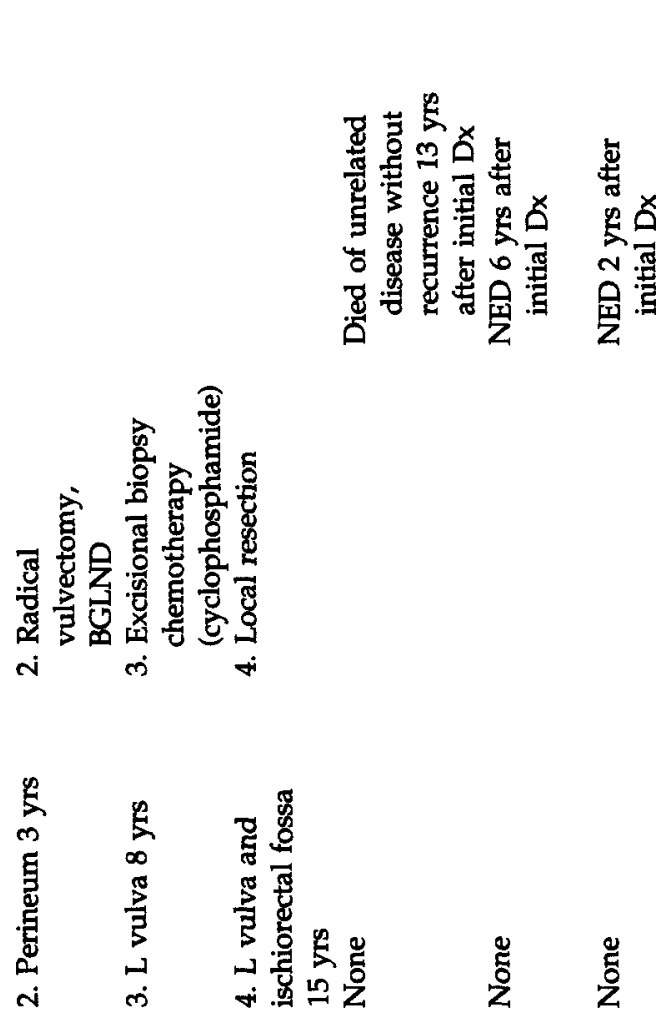

そั

ஜ્ર

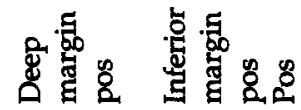

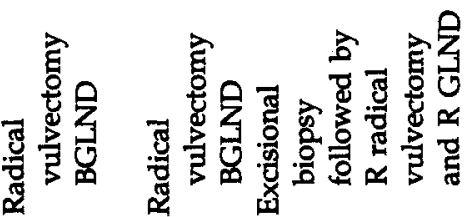

푤

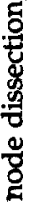

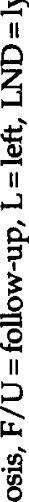

